\title{
RELACIONAMENTO DE PREÇOS NO MERCADO NORDESTINO DE TOMATE ${ }^{1}$
}

\author{
Rodrigo de Oliveira Mayorga ${ }^{2}$ \\ Francisco José Silva Tabosa \\ Ruben Dario Mayorga ${ }^{4}$ \\ Patrícia Verônica Pinheiro Sales Lima
}

Resumo: O objetivo deste artigo é analisar o relacionamento de preços do tomate entre os principais mercados atacadistas do Nordeste. Para isso, foram utilizados os seguintes métodos de séries temporais: teste de raiz unitária, co-integração de Johansen, modelo VAR, decomposição da variância dos erros de previsão e função impulso-resposta. Os resultados mostraram que o mercado atacadista de Fortaleza (CE) influencia os mercados de Ibiapaba (CE), Recife (PE) e Salvador (BA).

Palavras-chave: Mercado atacadista, transmissão de preços, Modelo VAR, tomate.

\section{Introdução}

Os estados da Bahia, Pernambuco e Ceará são os maiores produtores de tomates do Nordeste, e o bom desempenho destes estados pode ser atribuído à capacidade produtiva de seus solos, à disponibilidade de mãode-obra e à agricultura irrigada, tecnificada e competitiva.

Como consequência da elevada produção nordestina de tomates, notase o aumento no número de transações comerciais entre as regiões, de modo especial, entre Fortaleza (CE), Ibiapaba (CE), Recife (PE) e Salvador (BA). No entanto, ainda é desconhecida a relação de causalidade no processo de formação dos preços, ou a existência de

\footnotetext{
Recebido em 12/11/2008; Aceito em 10/03/2009.

Administrador de Empresas. Mestre em Economia Rural na UFC. E-mail: rsmayorga@ @otmail.com.

Economista. Doutorando em Economia no CAEN/ UFC e bolsista do CNPq. E-mail: franze@caen.ufc.br.

4 Economista. PhD. Professor Associado do Departamento de Economia Agrícola da UFC. E-mail: dario@ ufc.br.

5 Engenheira Agrônoma. Doutora Professora Adjunta do Departamento de Economia Agrícola da UFC. E-mail: pvpslima@ufc.br.
} 
integração nos mercados mencionados. Na revisão de literatura constatam-se trabalhos já realizados por diversos autores sobre integração de mercado para produtos agrícolas no Nordeste do Brasil (TABOSA et al. 2004; MAYORGA et al. 2007; TABOSA et al. 2008).

O presente estudo pretende agregar-se a estes e adota a hipótese de que existe relacionamento entre os preços do mercado produtor de Ibiapaba e os de diferentes mercados atacadistas. A rejeição dessa hipótese admite ausência de relacionamento entre os preços e, portanto, a existência de algumas imperfeições no mercado. Diante do exposto, acredita-se que um melhor entendimento acerca do relacionamento de preços no mercado nordestino do tomate pode ser útil à elaboração e implantação de políticas públicas que visem ao melhoramento do mercado privado e ao estímulo à competição nos mercados.

Os testes de estacionariedade e VAR, utilizados neste estudo, providenciam estimativas econométricas da natureza da integração entre mercados separados espacialmente. Modelos dessa natureza identificam a existência de imperfeições no mercado, mas não indicam quais das imperfeições citadas anteriormente podem estar presentes.

O objetivo deste estudo é analisar o relacionamento de preços entre os principais mercados atacadistas de tomate no Nordeste.

\section{Referencial Teórico}

\subsection{Integração de Mercados Agrícolas}

Pode-se definir mercados integrados como aqueles nos quais os preços de produtos diversos não se comportam independentemente, ou seja, é a expansão estável dos preços entre mercados em uma estação específica do ano, apesar das várias mudanças nos preços (DELGADO, 1986). Dessa forma, de acordo com Barret (1996), ocorrerá integração de mercados quando um choque nos preços de um deles provocar alterações nos preços do segundo. 
Rodrigo de Oliveira Mayorgá Francisco José Silva Tabosa, Ruben Dario Mayorga \& Patrícia Verônica Pinheiro Sales Lima

Conforme Santos et al. (2007), dentre os conceitos que descrevem a dependência entre mercados, destacam-se a arbitragem espacial e a Lei do Preço Único (LPU). Segundo Fackler e Gooldwin (2000), a arbitragem espacial se dá por intermédio de arbitradores que garantem que a diferença entre os preços de bens homogêneos, em duas regiões, seja consequência, no máximo, do custo de transferência do bem da região de menor preço para a de maior preço. A LPU, base analítica da integração de mercados, postula que bens homogêneos obedeçam à regra da perfeita arbitragem. Para Coelho (2002), no contexto da co-integração perfeita, integração de mercados e LPU são conceitos equivalentes.

Os primeiros pesquisadores do setor agrícola a estudar a transmissão de preços e a integração de mercado utilizaram, em sua maioria, análise de correlação de preços e regressão simples. Esses modelos, no entanto, passaram a ser criticados pela sua negligência, que mascara a presença de outros fatores que possam causar variações nos preços, como inflação, sazonalidade, crescimento populacional, problemas climáticos, entre outros (TIMMER, 1974; HARRIS, 1979 apud GOLETTI; RAISUDDIN; FARID, 1995, p. 188). Além disso, não havia o cuidado de verificar se as séries eram estacionárias.

Uma maneira de evitar essas críticas foi considerar a diferenciação de preços, que tem a propriedade atrativa de interpretar integração de mercado como interdependência de mudança de preços em diferentes mercados. Além disso, diferenciação de preço elimina a tendência comum que causa regressão espúria (GOLETTI et al. 1995, p. 188).

\section{Material e Métodos}

\section{1. Área Geográfica de Estudo e Origem dos Dados}

Os dados utilizados neste trabalho foram obtidos da Secretaria de Desenvolvimento Agrário do Estado do Ceará (SDA-CE). Consistem em séries de preços semanais de tomate, no período de janeiro de 2001 
a dezembro de $2005^{6}$, e correspondem aos mercados atacadistas de Fortaleza-CE, Ibiapaba-CE, Recife-PE e Salvador-BA. Os dados estão expressos em reais, por quilo $(\mathrm{R} \$ / \mathrm{kg})$.

As séries estimadas para análise de relação de preços do tomate, no mercado atacadista nordestino, foram: LFOR - logaritmo natural do preço de Fortaleza-CE; LIBI - logaritmo natural do preço da Ibiapaba-CE; LREC - logaritmo natural do preço de Recife-PE; e LSAL - logaritmo natural do preço de Salvador-BA. O software utilizado foi o Eviews v5.1.

Algumas considerações devem ser feitas no que diz respeito à deflação dos preços e ao deflator utilizado. De acordo com Pino e Rocha (1994, apud MARGARIDO, 1998, p. 71), sendo $z_{t}$ a série original nãodeflacionada e $d_{t}$, um deflator apropriado,

$$
y_{t}=\frac{z_{t}}{d_{t}}=Y_{t}=\log \frac{y_{t}}{y_{t-1}}=\log \frac{z_{t}}{z_{t-1}}-\log \frac{d_{t}}{d_{t-1}} .
$$

No entanto, é usual ajustar o modelo à série centrada ao redor da média, quando se toma uma diferença de ordem 1 (um): $Y_{t}-\bar{Y}$. Nesse caso,

$$
\bar{Y}=\frac{1}{n} \sum_{i} Y_{i}=\frac{1}{n} \sum \log \frac{z_{t}}{z_{t-1}}-\frac{1}{n} \sum \log \frac{d_{t}}{d_{t-1}} .
$$

Porém, se a taxa de inflação for semelhante mês a mês, no período considerado, então, qualquer que seja $t$, essa relação será aproximadamente constante. Logo, 6 A não-inclusão de séries mais recentes ocorreu devido à carência de dados confiáveis e à disponibilidade
destes, nos quatro mercados analisados. 
Rodrigo de Oliveira Mayorga' Francisco José Silva Tabosa, Ruben Dario Mayorga \& Patrícia Verônica Pinheiro Sales Lima

$\frac{d_{t}}{d_{t-1}} \approx k=\log \frac{y_{t}}{y_{t-1}}-\frac{1}{n} \sum \log \frac{y_{i}}{y_{i-1}}=Y_{t}-\bar{Y} \approx \log \frac{z_{t}}{z_{t-1}}-\frac{1}{n} \sum \log \frac{z_{i}}{z_{i-1}}$.

Portanto, da forma como as séries foram transformadas, o modelo com a série deflacionada é, aproximadamente, equivalente ao modelo com a série sem deflação.

\subsection{Teste de Raiz Unitária}

Para testar a presença, ou não, de raiz unitária na série e ordem de integração, foi usado o Teste de Dickey-Fuller Aumentado - ADF, desenvolvido por Dickey e Fuller (1981), que tem como base a seguinte expressão:

$$
\Delta Y_{t}=\beta+\delta T+\gamma Y_{t-1}+\alpha_{i} \sum_{i=1}^{m} \Delta Y_{t-i}+\varepsilon_{t}
$$

em que Y é a série de preços de tomate; $\beta$, constante; $\mathrm{T}$, tendência; e $\Delta$, operador de diferença, o qual é representado por $\Delta=\mathrm{Y}_{\mathrm{t}}^{\mathrm{t}}-\mathrm{Y}_{\mathrm{t}-1}{ }^{7}$

A hipótese nula é de que $\gamma=0$, ou seja, existe raiz unitária (isto é, a série é não-estacionária). A hipótese alternativa é que $\gamma<0$, ou seja, a série é estacionária.

O teste de ADF é, entretanto, muito sensível à presença de valores atípicos (FRANSES; HALDRUP (1994); CATI et al. (1999) apud FERNANDES; TORO (2005), p. 9). Nesse caso, utiliza-se o teste KPSS, desenvolvido por Kwiatkowski, Phillips, Schmidt e Shin (1992), que sugeriram um teste cuja principal característica é inversão das hipóteses em teste, isto é, a hipótese nula admite a noção de que a série é

A diferenciação de preços tem a propriedade atrativa de interpretar integração de mercado como interdependência de mudança de preços em diferentes mercados. Além disso, diferenciação de preço elimina a tendência comum que causa regressão espúria (GOLETTI et al. 1995, p. 188). Apesar de a estacionariedade poder ser alcançada pela diferenciação, nem sempre é uma solução satisfatória (PLOSSER; SCHWERT, 1978 apud ARDENI, 1998, p. 661). Além disso, em muitos casos, o que importa é a relação entre as variáveis em nível, o que seria perdido se as séries fossem diferenciadas. 
estacionária e, sob a hipótese alternativa, é integrada de ordem um. A vantagem desse teste resulta da falta de potência dos testes convencionais, pois estes tendem a não rejeitar a hipótese nula com demasiada frequência.

Seja $y_{t}, t=1,2, \ldots, T$, a série observada para a qual quer se testar a estacionariedade. Admitindo-se a noção de que possa se decompor a série na soma de tendência determinística $(\xi)$, passeio aleatório $(r)$ e erro estacionário $(\varepsilon)$ :

$y_{t}=\xi_{t}+r_{t}+\varepsilon_{t}$

$r_{t}=r_{t-1}+u_{t}$

sendo $u_{t} \sim \mathrm{iidN}\left(0, \sigma_{u}^{2}\right)$, processo independente e normalmente distribuído, o valor inicial de $r, r_{0}$ é considerado fixo e tem função de intercepto. A hipótese de estacionariedade é simplesmente $\sigma_{u}^{2}=0$, desde que se admita que $\varepsilon_{t}$ seja estacionário, sob a hipótese nula de $y_{t}$ ser estacionário em torno de uma tendência. Também se considera um caso especial do modelo (5), no qual se considera que $\xi=0$, sob a hipótese nula de $y_{t}$ ser estacionário em torno de um nível $\left(r_{0}\right)$, em vez de uma tendência.

A estatística utilizada é teste unilateral LM para a hipótese de $\sigma_{u}^{2}=0$, sob a pressuposição mais forte de que $u_{t}$ seja normal e que $\varepsilon_{t}$ seja iid $\mathrm{N}\left(0, \sigma_{u}^{2}\right)$. Os valores críticos para testar LM são baseados nos resultados assintóticos tabelados por KPSS.

De acordo com Kwiatkowski et al. (1992, p. 176), o teste KPSS tende a complementar o teste de raiz unitária, de Dickey-Fuller. Ao testar ambas as hipóteses, de raiz unitária e de estacionariedade, podem-se distinguir 
Rodrigo de Oliveira Mayorgá Francisco José Silva Tabosa, Ruben Dario Mayorga \& Patrícia Verônica Pinheiro Sales Lima

séries que aparentam ser estacionárias, que aparentam possuir raiz unitária e para as quais os dados (ou testes) não são suficientemente informativos para assegurar se são estacionárias ou integradas.

\subsection{Vetores Auto-regressivos (VAR)}

Para simplificar a análise foi utilizado um exemplo de sistema de equações com duas variáveis, as quais são interdependentes e também relacionadas com uma memória auto-regressiva (a sequência $X_{t}$ é afetada pelo seu passado e pela sequência $Z_{t}$, e vice-versa). A estacionariedade é uma condição fundamental para as propriedades dos estimadores do modelo. Analiticamente, conforme Enders (1995), pode-se representar o VAR:

$$
\begin{aligned}
& X_{t}=\alpha_{10}+\alpha_{11} X_{t-1}+\alpha_{12} Z_{t-1}+\varepsilon_{t 1} \\
& Z_{t}=\alpha_{20}+\alpha_{21} X_{t-1}+\alpha_{22} Z_{t-1}+\varepsilon_{t 2}
\end{aligned}
$$

Pode-se escrever o modelo VAR, em notação matricial, da forma:

$$
Y_{t}=\alpha+\Pi_{1} Y_{t-1}+\Pi_{2} Y_{t-2}+\ldots+\Pi_{p} Y_{t-p}+\varepsilon_{t},
$$

em que $Y_{t}$ é vetor $(n \times 1)$ auto-regressivo de ordem $p ; \alpha$, vetor ( $n \times 1)$ de interceptos; , matriz de parâmetros de $\operatorname{ordem}(n \times n)$; e $\varepsilon_{t}$, termo de erro $\varepsilon_{t} \sim N(0, \Omega)$.

Os coeficientes estimados a partir da equação (8) não levam em conta os relacionamentos entre as variáveis expressas no modelo VAR. Então, o caminho mais apropriado para avaliar os impactos das inovações é dado pela função impulso-resposta e pela decomposição de variância (MARGARIDO, 1998). 


\subsubsection{Função de Impulso-Resposta}

Uma função impulso-resposta delineia o comportamento das séries incluídas no modelo VAR, em resposta a choques ou a mudanças provocadas por variáveis residuais (ENDERS, op. cit.).

Considerando-se o modelo VAR (7), o efeito de um choque ou de uma mudança em $\varepsilon_{t 1}$ altera imediatamente os valores correntes da variável $X_{t}$, mas também os valores futuros de $X_{t}$ e $Z_{t}$, uma vez que os valores defasados $X_{t-1}$ aparecem nas duas equações.

Se as inovações $\varepsilon_{t 1}$ e $\varepsilon_{t 2}$ não fossem correlacionadas contemporaneamente, a interpretação da função impulso-resposta seria direta, e cada uma delas diretamente associada a uma variável, como aparece no modelo. Nesse caso, a função impulso-resposta, relativa à inovação $\varepsilon_{t 2}$, por exemplo, mediria o efeito de um choque sobre os valores correntes e futuros de $Z_{t}$ e sobre os valores futuros de $X_{t}$.

\subsubsection{Decomposição da Variância}

De acordo com Enders (op. cit.), a decomposição de variância fornece o percentual do erro da variância prevista atribuída aos choques de determinada variável versus os choques nas outras variáveis do sistema. Se os choques observados numa variável $z$ não forem capazes de explicar a variância do erro de previsão da variável $y$, diz-se que a sequiência $y$ é exógena. Caso contrário, diz-se que ela é endógena.

A decomposição da variância dos erros de previsão permite separar a variância dos erros de previsão para cada variável em componentes que podem ser atribuídos por ela própria e pelas demais variáveis endógenas, 
Rodrigo de Oliveira Mayorgá Francisco José Silva Tabosa, Ruben Dario Mayorga \& Patrícia Verônica Pinheiro Sales Lima

isoladamente, apresentando, em termos percentuais, qual o efeito que um choque não antecipado sobre determinada variável tem sobre ela própria e sobre as demais variáveis pertencentes ao sistema (MARGARIDO, op. cit.).

\subsection{Vetor de Correção de Erros (VEC)}

A condição necessária para que os estimadores obtidos possuam propriedades desejáveis é que as variáveis do VAR sejam estacionárias. Caso contrário, a existência de raízes unitárias deve ser levada em consideração.

De acordo com Johnston e Dinardo (1997, p. 301), quando as variáveis no modelo VAR são integradas de primeira ordem ou mais, estão sujeitas às inconsistências de regressões, considerando que as variáveis sejam não-estacionárias.

\subsubsection{Testes de Co-integração de Johansen}

Retoma-se a equação (8), do modelo VAR, em notação matricial, no entanto, considerando-se que $Y_{t}$ seja um vetor com $n$ variáveis $(n \mathrm{x} 1)$, $n \geq 2$, supondo que sejam integrados de ordem $1, I(1)$, não estacionárias. $\mathrm{O}$ vetor pode ser expresso por:

$$
Y_{t}=\alpha+\Pi_{1} Y_{t-1}+\Pi_{2} Y_{t-2}+\ldots+\Pi_{p} Y_{t-p}+\varepsilon_{t}
$$


A equação (9) pode ser modificada em um Modelo de Correção de Erros, cujo formato é:

$$
\Delta Y_{t}=\Pi Y_{t-1}+\Gamma_{1} \Delta Y_{t-1}+\ldots+\Gamma_{p-1} \Delta Y_{t-p+1}+\varepsilon_{t}
$$

em que $\Pi=\Pi_{1}+\Pi_{2}+\ldots+\Pi_{p}-I ; i=1,2,3, \ldots, p-1$

$$
\Gamma_{i}=-\sum_{j=i+1}^{p} \Pi j
$$

\section{$I=$ matriz identidade}

A matriz $\Pi(n \times n)$ pode ser vista com maior nível de detalhe, sendo representada por:

$\Pi=\alpha \beta^{\prime}$,

em que $\alpha$ é matriz que representa a velocidade de ajustamento dos parâmetros no curto prazo; $\beta$, matriz de coeficientes de co-integração, no longo prazo, entre as variáveis. Os parâmetros $\alpha$ e $\beta$ são matrizes de dimensão $n \times r$, em que $n$ é o número de variáveis incluídas no modelo e $r$, número de vetores de co-integração da matriz $\Pi$.

Podem-se ter, então, os seguintes casos (PATTERSON, 2000, p. 620): Se todos os autovalores de $\Pi$ forem diferentes de zero (isto é, $r=n$, colunas linearmente independentes), esta matriz terá posto completo $\Pi(1)=\Pi_{1}+\ldots+\Pi_{p}$, o que implica que todos os componentes de $Y_{t}$ serão estacionários e a representação válida será o VAR (p), em nível dado pela equação (9); se todos os autovalores de $\Pi$ forem iguais a zero (isto é, $r=0$ ), esta matriz será, portanto, indistinguível da matriz nula, o que implica também que os componentes de $Y_{t}$ serão, no mínimo, $I(1)$, e a representação válida será um VAR (p-1) em primeira diferença, isto é, equação (10) sem o termo em nível; se $\Pi$ tiver posto reduzido, isto é, $0<r<n$, nesse caso, ter-se-ão $n-r$ autovalores diferentes de zero. 
Os componentes de $Y_{t}$ serão, no mínimo, $I(1)$, e a representação válida será a equação (10) com $\Pi=\alpha \beta^{\prime}$, em que $\alpha$ e $\beta$ são matrizes $n \times r$, de posto $r$. Essa representação é chamada Vetor de Correção de Erros (VEC) e nela estão presentes $r$ relações de co-integração.

Johansen e Juselius (1990) desenvolveram dois testes capazes de determinar o posto da matriz $\Pi$, da equação (11). O primeiro, conhecido como teste traço, é dado por:

$$
\lambda_{\text {trace }}=-T \sum_{i=r+1}^{n} \ln (1-\hat{\lambda})
$$

em que $r=0,1,2, \ldots, n-2, n-1$ e $\hat{\lambda}$ é valor estimado dos autovalores obtidos da matriz $\beta ; T$, número de observações.

O teste traço avalia a hipótese nula de que o número de vetores diferentes de co-integração seja menor ou igual a $r$, contra uma hipótese geral.

$$
H_{o}: \lambda=0 \quad i=r+1, \ldots, n
$$

A não-rejeição de $H_{o}$ indica presença de, no máximo, $r$ vetores de cointegração. Se $H_{o}$ for rejeitada, deve-se repetir o teste para $r+1$ e determinar se existem vetores de co-integração.

O segundo teste é o do máximo autovalor, que testa a existência de exatamente $r$ vetores de co-integração, contra a alternativa de existência de $r+1$ vetores.

$$
\lambda_{\max }=-T \ln \left(1-\hat{\lambda}_{r+1}\right),
$$


em que a hipótese nula dada por:

$H_{o}: \lambda_{r+1}=0$.

A não-rejeição de $H_{o}$ indica presença de exatamente $r$ vetores de cointegração.

A inclusão de termos deterministas também é essencial para correta implantação do procedimento de Johansen. Pode-se representar a inclusão desses termos em (11) por:

$\Delta Y_{t}=\Pi Y_{t-1}+\Gamma_{1} \Delta Y_{t-1}+\ldots+\Gamma_{p-1} \Delta Y_{t-p+1}+\Phi D_{t}+\varepsilon_{t}$

em que $D_{t}$ pode representar tanto uma constante como uma tendência e, ou, uma variável dummy. A escolha dos termos deterministas deve ser feita com auxílio de uma inspeção visual nos dados e também mediante testes apropriados sobre a significância dos termos deterministas. A determinação correta do número de defasagens é fundamental para análise de co-integração, ou seja, o valor de $p$, em (14), pode ser obtido por vários métodos, entre eles, os de Akaike Information Criterion (AIC), Schwarz (SIC), e Hannan-Quinn (HQ).

\section{Resultados e Discussão}

O primeiro passo da análise consistiu na realização dos testes de raiz unitária. Para isso, aplicou-se o Dickey-Fuller Aumentado (ADF) para verificar a estacionariedade das séries, com defasagens baseadas no AIC, obtendo-se os resultados apresentados na Tabela 1. As estatísticas $\tau_{t}, \tau_{\mu}, \tau$ correspondem, respectivamente, às equações com constante e com tendência; com constante e sem tendência; e sem constante e sem tendência. 
Rodrigo de Oliveira Mayorga' Francisco José Silva Tabosa, Ruben Dario Mayorga \& Patrícia Verônica Pinheiro Sales Lima

Tabela 1 - Teste de Raiz Unitária e de Dickey-Fuller Aumentado (ADF), para as séries de preços logarítmizadas em níveis, janeiro de 2001 a dezembro de 2005

\begin{tabular}{lcccccc}
\hline & $\tau_{t}$ & Defasagens & $\tau_{\mu}$ & defasagens & $\tau$ & defasagens \\
\hline LFOR & $-4,8780^{*}$ & 10 & $-4,2321^{*}$ & 10 & $-4,1040^{*}$ & 1 \\
LIBI & $-5,8530^{*}$ & 0 & $-5,5737^{*}$ & 0 & $-3,3358^{*}$ & 2 \\
LREC & $-4,8463^{*}$ & 5 & $-4,8607^{*}$ & 5 & $-4,1707^{*}$ & 5 \\
LSAL & $-5,0462^{*}$ & 9 & $-3,7641^{*}$ & 9 & $-3,1938^{*}$ & 3 \\
\hline
\end{tabular}

Os valores críticos para o modelo com constante e com tendência são $-3,9945,-3,4276$ e -3,1371, a $1 \%, 5 \%$ e $10 \%$, respectivamente; para o modelo com constante e sem tendência, $-3,4559,-2,8727$ e 2,5728 , a $1 \%, 5 \%$ e $10 \%$, respectivamente; e para o modelo sem constante e sem tendência, -2,5737, 1,9420 e $-1,6158$, a $1 \%, 5 \%$ e $10 \%$, respectivamente.

***indica que a hipótese nula é rejeitada a $10 \%$.

**indica que a hipótese nula é rejeitada a $5 \%$.

*indica que a hipótese nula é rejeitada a $1 \%$.

Fonte: Dados da pesquisa.

Verificou-se que todas as séries foram estacionárias, a 1\% de significância, nos três modelos de equações. Tendo em vista que o teste ADF é muito sensível à presença de valores atípicos, fez-se necessária a estimação dos testes de raiz unitária com presença de quebras. Na Tabela 2 são apresentados os resultados dos testes de estacionariedade formulados por Kwiatkowski, Phillips, Schmidt e Shin (1992) - KPSS.

Tabela 2 - Teste de estacionariedade formulado por Kwiatkowski-PhillipsSchmidt-Shin, para as séries de preços logaritmizadas em níveis, janeiro de 2001 a dezembro de 2005

\begin{tabular}{llclc}
\hline & Tendência e Constante & defasagens & Constante & defasagens \\
\hline LFOR & 0,0989 & 11 & $0,8138^{*}$ & 11 \\
LIBI & 0,0505 & 11 & $0,5579^{* *}$ & 11 \\
LREC & $0,0,277$ & 11 & 0,0382 & 11 \\
LSAL & 0,0436 & 11 & $1,0491^{*}$ & 11 \\
\hline
\end{tabular}

Os valores críticos para o modelo com constante e com tendência são $0,2160,0,1460$ e 0,1190 , a $1 \%, 5 \%$ e $10 \%$, respectivamente; e para o modelo com constante e sem tendência, $0,7390,0,4630$ e 0,3470 , a $1 \%, 5 \%$ e $10 \%$, respectivamente.

***indica que a hipótese nula é rejeitada a $10 \%$.

**indica que a hipótese nula é rejeitada a $5 \%$.

*indica que a hipótese nula é rejeitada a $1 \%$.

Fonte: Dados da pesquisa. 
As séries LFOR, LIBI e LSAL foram estacionárias com tendência e com constante. No entanto, com constante e sem tendência, as séries sinalizaram rejeição da hipótese nula a $1 \%, 5 \%$ e $1 \%$ de significância, respectivamente. A série LREC mostrou-se estacionária nos modelos com constante e com tendência e com constante e sem tendência. Portanto, realizou-se o teste KPSS para as séries na primeira diferença. Os resultados estão apresentados na Tabela 3.

Tabela 3 - Teste de estacionariedade, formulado por KwiatkowskiPhillips-Schmidt-Shin, para as séries de preços logaritmizadas na primeira diferença, janeiro de 2001 a dezembro de 2005

\begin{tabular}{llclc}
\hline & Tendência e Constante & defasagens & Constante & defasagens \\
\hline D(LFOR) & 0,0267 & 14 & 0,0458 & 14 \\
D(LIBI) & 0,0270 & 12 & 0,0494 & 12 \\
D(LREC) & 0,0321 & 17 & 0,0435 & 17 \\
D(LSAL ) & 0,0903 & 42 & 0,0914 & 41 \\
\hline
\end{tabular}

Os valores críticos para o modelo com constante e com tendência são $0,2160,0,1460$ e 0,1190 , a $1 \%, 5 \%$ e $10 \%$, respectivamente; e para o modelo com constante e sem tendência, $0,7390,0,4630,0,3470$, a $1 \%, 5 \%$ e $10 \%$, respectivamente.

***indica que a hipótese nula é rejeitada a $10 \%$.

**indica que a hipótese nula é rejeitada a $5 \%$.

*indica que a hipótese nula é rejeitada a $1 \%$.

Fonte: Dados da pesquisa.

Os testes apontam que todas as séries analisadas foram estacionárias na primeira diferença, em ambos os modelos, o que indica que as variáveis são estacionárias nas diferenças. Dessa maneira, podem ser consideradas como integradas de ordem um.

Antes de realizar o teste de co-integração, foi necessário determinar o número de defasagens a serem utilizadas e escolher o modelo a ser empregado. O critério de informação usado para determinação do número de defasagens foi o de Akaike (AIC), que apresentou menor valor para defasagem de ordem um ${ }^{8}$.

8 Optou-se pelo critério AIC, segundo Margarido (1998), procurando estimar um modelo que captasse a maior quantidade de informações contidas entre as variáveis. Ver anexo. 
Rodrigo de Oliveira Mayorgá Francisco José Silva Tabosa, Ruben Dario Mayorga \& Patrícia Verônica Pinheiro Sales Lima

Os resultados dos testes de co-integração, apresentados na Tabela 4, mostram que a hipótese nula de não co-integração foi rejeitada, visto que o valor calculado da estatística traço $\left(\lambda_{\text {trace }}\right)$ foi igual a 133,020, superior ao seu respectivo valor crítico ao nível de $1 \%(60,16)$. Portanto, concluiuse que há quatro vetores de co-integração, já que a hipótese nula de que existem até três vetores co-integrados foi rejeitada, pois o valor calculado $(12,70)$ para a estatística $\left(\lambda_{\text {trace }}\right)$ foi superior ao seu respectivo valor crítico $(9,24)$, a $5 \%$. Como o número de vetores de co-integração é igual ao número de variáveis, ou seja, o rank é pleno, deve-se utilizar o Modelo Vetorial Auto-Regressivo (VAR) em nível.

Tabela 4 - Resultado do teste de co-integração de Johansen, variáveis LFOR, LIBI, LREC, LSAL, janeiro de 2001 a dezembro de 2005

\begin{tabular}{ccclcc}
\hline Eigenvalue & $\begin{array}{c}\text { Hipótese } \\
\text { Nula }\end{array}$ & $\begin{array}{c}\text { Hipótese } \\
\text { Alternativa }\end{array}$ & $\begin{array}{c}\text { Estatística traço } \\
\lambda_{\text {trace }} \text { calculado }\end{array}$ & $\begin{array}{c}\text { Estatística traço } \\
\lambda_{\text {trace }} \text { valor } \\
\text { crítico ao nível de } \\
5 \% .\end{array}$ & $\begin{array}{c}\text { Estatística traço } \\
\lambda_{\text {trace valor }} \\
\text { crítico ao nível } \\
\text { de } 1 \% .\end{array}$ \\
\hline 0,2100 & $r=0$ & $r>0$ & $133,020^{*}$ & 53,12 & 60,16 \\
0,1294 & $r \leq 1$ & $r>1$ & $71,234^{*}$ & 34,91 & 41,07 \\
0,0812 & $r \leq 2$ & $r>2$ & $34,907^{*}$ & 19,96 & 24,60 \\
0,0473 & $r \leq 3$ & $r>3$ & $12,702^{* *}$ & 9,24 & 12,97 \\
\hline
\end{tabular}

$* *$ indica que a hipótese nula é rejeitada a $5 \%$.

*indica que a hipótese nula é rejeitada a $1 \%$.

Fonte: Dados da pesquisa.

A Tabela 5 apresenta os resultados da decomposição da variância dos erros de previsão, para quatro variáveis. A ordenação escolhida foi LFOR, LIBI, LREC e LSAL, já que, conforme CEASA (2004), a CEASA de Fortaleza (CE) é a central com maior volume de comercialização no Nordeste. A primeira coluna determina a variável atribuída a um choque não antecipado, enquanto a segunda representa os períodos, no presente trabalho, expressos em semanas. Considera-se também que um choque não antecipado sobre as variáveis analisadas perdure no máximo por 24 semanas. No caso da variável LFOR, a terceira coluna informa o 
percentual da variância dos erros de previsão em função de choques não antecipados sobre essa variável. As demais colunas captam percentuais das variâncias dos erros de previsão de LFOR, atribuídos às variáveis LIBI, LREC e LSAL.

Tabela 5 - Decomposição da variância dos erros de previsão, em percentagem de LFOR, para as variáveis LIBI, LREC e LSAL. Janeiro de 2001 a dezembro de 2005

\begin{tabular}{lccccc}
\hline Variável & Período & LFOR & LIBI & LREC & LSAL \\
LFOR & 1 & 100,000 & 0,000 & 0,000 & 0,000 \\
& 6 & 94,110 & 4,001 & 0,347 & 1,540 \\
& 12 & 93,580 & 3,930 & 0,474 & 2,014 \\
& 18 & 93,443 & 3,865 & 0,550 & 2,140 \\
& 24 & 93,411 & 3,854 & 0,580 & 2,165 \\
\hline
\end{tabular}

Fonte: Dados da pesquisa.

Os resultados da decomposição da variância dos erros de previsão de LFOR mostraram que, decorridas 24 semanas após um choque não antecipado sobre essa variável, $93,41 \%$ de seu comportamento decorreu dela própria e $6,59 \%$, das variáveis LIBI $(3,85 \%)$, LREC $(0,58 \%)$, LSAL $(2,16 \%)$

Em relação à LIBI, grande parte de seu próprio erro de previsão é explicada pela variável LFOR, responsável por cerca de 64,23\%, decorridos 24 meses após um choque inicial não antecipado. As variáveis LREC e LSAL foram responsáveis por 2,65\% do erro de previsão, e cerca de $33,11 \%$ refere-se a ela própria (Tabela 6). 
Rodrigo de Oliveira Mayorga' Francisco José Silva Tabosa, Ruben Dario Mayorga \& Patrícia Verônica Pinheiro Sales Lima

Tabela 6 - Decomposição da variância dos erros de previsão, em percentagem de LIBI, para as variáveis LFOR, LREC e LSAL. Janeiro de 2001 a dezembro de 2005

\begin{tabular}{lccccc}
\hline Variável & Período & LFOR & LIBI & LREC & LSAL \\
LIBI & 1 & 42,237 & 57,762 & 0,000 & 0,000 \\
& 6 & 61,551 & 36,152 & 0,746 & 1,548 \\
& 12 & 63,929 & 33,550 & 0,759 & 1,759 \\
& 18 & 64,201 & 33,166 & 0,799 & 1,833 \\
& 24 & 64,234 & 33,107 & 0,809 & 1,848 \\
\hline
\end{tabular}

Fonte: Dados da pesquisa.

Os resultados da decomposição da variância dos erros de previsão de LREC, Tabela 7, mostraram que, decorridas 24 semanas após um choque não antecipado sobre essa variável, $50,18 \%$ de seu comportamento decorreu dela própria, e os $50 \%$ restantes são atribuídos, principalmente, a LFOR $(29,13 \%)$ e LIBI $(19,92 \%)$, enquanto a variável LSAL representou apenas $0,77 \%$.

Tabela 7 - Decomposição da variância dos erros de previsão, em percentagem de LREC, para as variáveis LIBI, LLFOR e LSAL. Janeiro de 2001 a dezembro de 2005

\begin{tabular}{lccccc}
\hline Variável & Período & LFOR & LIBI & LREC & LSAL \\
LREC & 1 & 6,676 & 1,889 & 91,433 & 0,000 \\
& 6 & 24,388 & 18,351 & 56,499 & 0,760 \\
& 12 & 28,499 & 19,982 & 50,751 & 0,766 \\
& 18 & 29,059 & 19,942 & 50,232 & 0,765 \\
& 24 & 29,130 & 19,923 & 50,175 & 0,770 \\
\hline
\end{tabular}

Fonte: Dados da pesquisa.

Para a variável LSAL, Tabela 8, após 24 semanas de um choque não antecipado sobre essa variável, $41,43 \%$ da sua decomposição da variância dos erros de previsão decorreu dela mesma, e os 58,57\% restantes, das outras variáveis. Verificou-se no entanto que, desses $58,57 \%$, LFOR respondeu por cerca de $53,57 \%$. 
Tabela 8 - Decomposição da variância dos erros de previsão em percentagem de LSAL, para as variáveis LIBI, LREC e LFOR. Janeiro de 2001 a dezembro de 2005

\begin{tabular}{lccccc}
\hline Variável & Período & LFOR & LIBI & LREC & LSAL \\
LSAL & 1 & 12,135 & 2,302 & 0,878 & 84,683 \\
& 6 & 46,498 & 3,482 & 1,526 & 48,491 \\
& 12 & 52,712 & 3,631 & 1,372 & 42,283 \\
& 18 & 53,475 & 3,581 & 1,411 & 41,532 \\
& 24 & 53,575 & 3,569 & 1,425 & 41,429 \\
\hline
\end{tabular}

Fonte: Dados da pesquisa.

Para a função impulso-resposta, Figura 1, a ordenação foi obtida pelo procedimento de Cholesky. Os resultados mostraram que o comportamento da função resposta de impulso sobre o preço de Fortaleza teve impacto bem definido no preço de Ibiapaba, dado que um choque no desvio-padrão dos preços de Fortaleza tendeu a elevar o desvio-padrão dos preços de Ibiapaba.

Após a incidência desse choque no desvio-padrão do preço de Ibiapaba, ele decaiu continuamente até a vigésima semana, quando tendeu a se dissipar. Isso ocorreu porque Ibiapaba é um mercado produtor, enquanto Fortaleza, além de ser um mercado atacadista, é o maior consumidor de tomates da Ibiapaba. Os preços de Recife e Salvador, após um choque não antecipado sobre o desvio-padrão do preço de Fortaleza, apresentaram comportamento semelhante, ou seja, inicialmente, notou-se elevação no desvio-padrão no nível de preços até a terceira ou quarta semana, quando atingiu o ponto máximo.

A partir desse ponto, os desvios-padrão dos preços tenderam a cair até a vigésima semana, quando tenderam a se dissipar, devido ao fato de que, quando o preço do tomate de Ibiapaba aumentava (ou pelo período de entressafra ou pelo aumento da demanda de Teresina-PI e/ou São LuísMA), a CEASA de Fortaleza procurava também ser abastecida pelas CEASAS de Recife e, ou, de Salvador, até uma queda no preço do tomate da Ibiapaba. 
Rodrigo de Oliveira Mayorgá Francisco José Silva Tabosa, Ruben Dario Mayorga \& Patrícia Verônica Pinheiro Sales Lima

No caso da variável LIBI, observou-se que um choque no desvio-padrão dessa variável causou pequena elevação no desvio-padrão do preço de Fortaleza, até a segunda semana após a incidência desse choque. Posteriormente, o desvio-padrão no preço de Fortaleza declinou amenamente, até a décima segunda semana, após o choque inicial (comportamento de mercado analisado no parágrafo anterior).

A variável LREC teve aumento no desvio-padrão dos preços, após um choque não antecipado sobre o desvio-padrão do preço de Ibiapaba. Essa elevação no desvio-padrão dos preços ocorreu até, aproximadamente, a terceira semana e, a partir desse ponto, houve reversão dessa tendência. Notou-se, então, queda lenta até estabilizarse, entre a décima quarta e a décima sexta semana (comportamento de mercado analisado no parágrafo anterior). 
Response of LIBI to LFOR

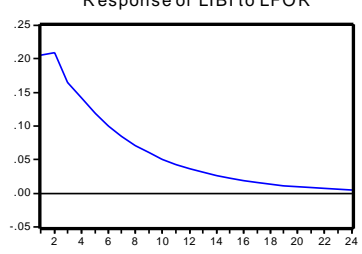

Response of LREC to LFOR

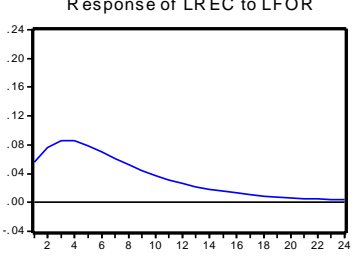

Response of LSAL to LFOR

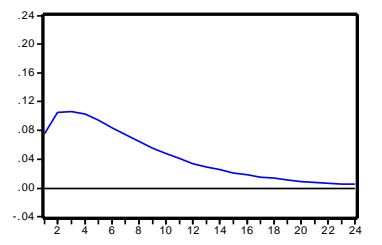

Response to Cholesky One S.D. Innovations

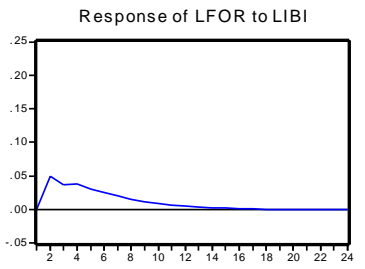

Response of LREC to LIBI

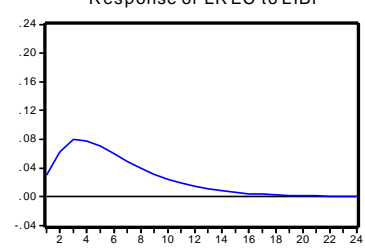

Response of LSAL to LIBI

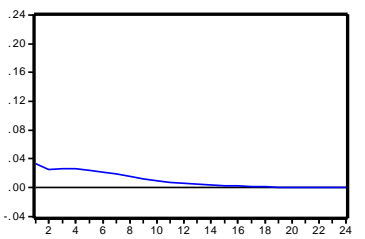

Response of LFOR to LREC

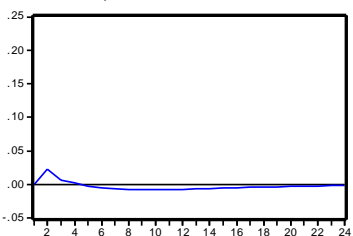

Response of LIBI to LREC

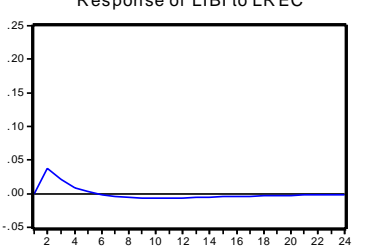

Response of LSAL to LREC

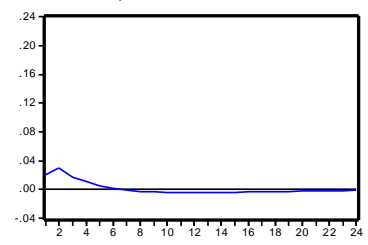

Response of LFOR to LSAL

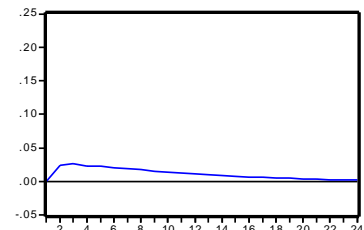

Response of LIBI to LSAL

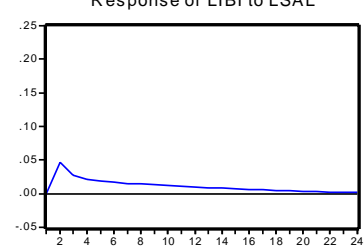

Response of LREC to LSAL

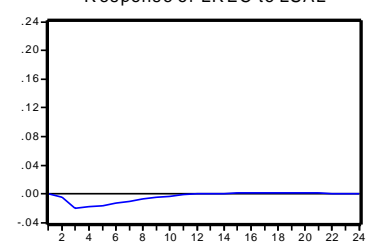

Fonte: Resultados da Pesquisa.

Figura 1 - Elasticidade de função de Resposta de Impulso, efeitos de choques em LFOR, LIBI, LREC e LSAL sobre LFOR, LIBI, LREC e LSAL. 
Rodrigo de Oliveira Mayorgá Francisco José Silva Tabosa, Ruben Dario Mayorga \& Patrícia Verônica Pinheiro Sales Lima

Já a relação entre LIBI e LSAL apresenta indícios de fraca integração na comercialização do produto entre os dois mercados, pois nesses mercados, segundo o CEASA (2004), fatores como distância, má infraestrutura das estradas e acesso a aeroportos dificultam a existência de integração. Nesse caso, pode-se incorporar a Arbitragem Espacial e a Lei do Preço Único (LPU), pois o custo de transação entre os dois mercados é grande, maior do que a diferença entre seus preços.

Verificou-se, também, que um choque não antecipado sobre os preços de Recife e Salvador não teve efeito significativos sobre as outras variáveis.

\section{Conclusões e Sugestões}

A análise do relacionamento de preços entre os principais mercados atacadistas de tomate no Nordeste apontou que e a maior parcela da decomposição da variância dos preços em Ibiapaba, Recife e Salvador foi decorrente de choques ocorridos no preço de Fortaleza. Os preços nessas cidades foram influenciados, mesmo decorridas 24 semanas, de um choque não antecipado nos preços em Fortaleza. Portanto, apresentam uma "memória" longa, especialmente os preços de Ibiapaba.

As funções impulso-resposta permitiram concluir que os preços em Fortaleza tiveram efeito sobre os preços em Ibiapaba, Recife e Salvador. A hipótese adotada neste estudo não foi rejeitada, uma vez que se contatou influência bem definida de Ibiapaba no mercado de Recife, embora nos de Fortaleza e Salvador não tenha havido impacto considerável. A interdependência de preços, nas diferentes cidades nordestinas analisadas, evidencia a presença de um mercado integrado regionalmente. 


\section{Referências}

ARDENI, P.G. Does the law of one price really hold? American Journal of Agricultural Economics, V. 71, N. 3, p. 661-669, Aug. 1998.

CEASA - Central de Abastecimento S/A. SIMA - Sistema de Informação de Mercado Agrícola. Preço médio mensal, 2000; 2001; 2002; 2003 e 2004

COELHO, A.B. A cultura do Algodão e a questão da Integração entre preços internos e externos. 136p. Dissertação (Mestrado em Economia) - Departamento de Economia da Faculdade de Economia, Administração e Contabilidade da Universidade de São Paulo, São Paulo, 2002.

DELGADO, C.L. A variance components approach to foodgrain market integration in North Nigeria. American Journal of Agricultural Economics, V. 68, N. 4, p. 970-979, Nov. 1986.

DICKEY, D.A.; FULLER, W.A. Likelihood ratio statistics for autoregressive time series with a unit root. Econometrica, V. 49, N. 4, p. 1057-1072, Jul. 1981.

ENDERS, W. Applied econometric time series. New York: John Wiley and Sons, 1995.

FACKLER, P.L.; GOODWIN, B.K. Spatial price analysis. forthcoming, Handbook of Agricultural Economics. North-Holland: 2000. p.1-59.

FERNANDES, M.; TORO, J. O Mecanismo Monetário de transmissão na Economia Brasileira Pós-Plano Real. Revista Brasileira de Economia, V. 59, N. 1, p. 5-32, Jan./Mar. 2005. Disponível em: <epge.fgv.br/portal/arquivo/1293.pdf> Acesso em: 15 jun. 2006. 
Rodrigo de Oliveira Mayorgá Francisco José Silva Tabosa, Ruben Dario Mayorga \& Patrícia Verônica Pinheiro Sales Lima

GOLETTI, F.; RAISUDDIN, A.; FARID, N. Structural determinants of market integration. The case of rice markets in Bangladesh. The Developing Economies, V. 33, N. 2, p. 185-202, Jun. 1995.

JOHANSEN, S.; JUSELIUS, K. Maximum Likelihood estimation and inference on cointegration with applications to the demand for money. Oxford Bulletin of Economics and Statistics, V. 52, N. 2, p. 169-210, 1990.

JOHNSTON, J.; DINARDO, J. Econometric Methods. 4 ed. Singapore: McGraw-Hill. 1997.

KWIATKOWSKI, D.; PHILLIPS, P.C.B.; SCHMIDT, P.; SHIN, Y.. Testing the null hypothesis of stationarity against the alternative of a unit root. How sure are we that economic time series have a unit root? NorthHolland: Journal of Econometrics, V. 54, p. 159-178. 1992.

MAYORGA, R. de O ; KHAN, Ahmad Saeed ; MAYORGA, R. D. ; LIMA, Patrícia Verônica Pinherio Sales ; MARGARIDO, M.A. . Análise de transmissão de preços do mercado atacadista de melão do Brasil. Revista de Economia e Sociologia Rural, v. 45, p. 275-704, 2007.

MARGARIDO, M.A. Transmissão de preços internacionais de suco de laranja para preços ao nível de produtor de laranja no Estado de São Paulo. Instituto de Economia Agrícola. Coleção Estudos Agrícolas 6. São Paulo-SP. 1998.

PATTERSON, K.D. An Introduction to Applied Econometrics: a time series approach. Great Britain: St. Martin's Press. 2000.

ROSADO, P.L. Integração espacial entre os mercados brasileiros de suínos. 117 p. (Tese de doutorado). Universidade Federal de Viçosa. Viçosa. Minas Gerais. 2006. 
SANTOS, V. F; PEREIRA, M. W. G.; BRAGA, M. J.; VIEIRA, W. C. Análise do preço do milho nos mercados externo e interno. Revista de Economia Agrícola. no 3, Jul./Ago./Set. 2007.

TABOSA, F. J. S. ; CASTELAR, L.I. de M. ; LINHARES, F. . Integração do Mercado Atacadista de Maracujá no Nordeste Brasileiro. In: V Encontro Economia do Ceará em Debate, 2008, Fortaleza. Anais do V Encontro Economia do Ceará em Debate, 2008. 12p.

TABOSA, F. J. S.; SILVA, D.M.F. ; MADALOZZO, C.L. ; CAMPOS, R.T . Análise Econométrica do Mercado do Tomate no Estado do Ceará. In: XLII Congresso da Sociedade Brasileira de Economia e Sociologia Rural - SOBER, 2004, Cuiabá-MT. Anais do XLII Congresso da Sociedade Brasileira de Economia e Sociologia Rural - SOBER. Brasília: SOBER, 2004., 8p.

\begin{abstract}
The objective this paper is to analyze the relationships in the prices on the tomatoes wholesale market prices of Fortaleza (CE), Ibiapaba (CE), Recife (PE) e Salvador (BA). Time series methods were used: unit root test, Johansen co-integration test, VAR models, and variance decomposition of prediction error and impulse response function. The results showed that the prices of Fortaleza influencies the wholesale market of Ibiapaba (CE), Recife (PE) e Salvador (BA).
\end{abstract}

Keywords: Wholesale market, price transmission, VAR model, tomatoes. 
Rodrigo de Oliveira Mayorga' Francisco José Silva Tabosa, Ruben Dario Mayorga \& Patrícia Verônica Pinheiro Sales Lima

\section{Anexo}

VAR Lag Order Selection Criteria

Endogenous variables: LFOR LIBI LREC LSAL

Exogenous variables: C

Sample: 11/01/2000 12/28/2005

Included observations: 257

\begin{tabular}{ccccccc}
\hline \hline Lag & LogL & LR & FPE & AIC & SC & HQ \\
\hline \hline 0 & -157.8977 & NA & $4.14 \mathrm{e}-05$ & 1.259905 & 1.315143 & 1.282119 \\
1 & 140.1849 & 584.5667 & $4.61 \mathrm{e}-06^{*}$ & $-0.935291^{*}$ & $-0.659098^{*}$ & $-0.824220^{*}$ \\
2 & 154.7344 & 28.08009 & $4.67 \mathrm{e}-06$ & -0.924003 & -0.426857 & -0.724075 \\
3 & 167.6053 & 24.43967 & $4.78 \mathrm{e}-06$ & -0.899652 & -0.181551 & -0.610868 \\
4 & 180.3638 & 23.82904 & $4.91 \mathrm{e}-06$ & -0.874426 & 0.064629 & -0.496785 \\
5 & 194.2990 & 25.59302 & $4.99 \mathrm{e}-06$ & -0.858358 & 0.301652 & -0.391859 \\
6 & 209.9848 & $28.31993^{*}$ & $5.01 \mathrm{e}-06$ & -0.855913 & 0.525051 & -0.300558 \\
7 & 220.7157 & 19.04001 & $5.22 \mathrm{e}-06$ & -0.814908 & 0.787010 & -0.170696 \\
8 & 230.9218 & 17.79117 & $5.47 \mathrm{e}-06$ & -0.769819 & 1.053053 & -0.036750 \\
\hline \hline
\end{tabular}

* indicates lag order selected by the criterion

LR: sequential modified LR test statistic (each test at 5\% level)

FPE: Final prediction error

AIC: Akaike information criterion

SC: Schwarz information criterion

HQ: Hannan-Quinn information criterion 
REVISTA DE ECONOMIA E AGRONEGÓCIO, VOL.7, $N^{\circ} 1$ 